Voix et Images

voixetimages

\title{
Le monologue intérieur dans The Sound and the Fury de William Faulkner et le Sourd dans la ville de Marie-Claire Blais
}

\section{Martin Herden}

Volume 14, numéro 3 (42), printemps 1989

Gabrielle Roy

URI : https://id.erudit.org/iderudit/200801ar

DOI : https://doi.org/10.7202/200801ar

Aller au sommaire du numéro

Éditeur(s)

Université du Québec à Montréal

ISSN

0318-9201 (imprimé)

1705-933X (numérique)

Découvrir la revue

\section{Citer cet article}

Herden, M. (1989). Le monologue intérieur dans The Sound and the Fury de William Faulkner et le Sourd dans la ville de Marie-Claire Blais. Voix et Images, 14(3), 483-496. https://doi.org/10.7202/200801ar 


\title{
Le monologue intérieur dans The Sound and the Fury de William Faulkner et le Sourd dans la ville de Marie-Claire Blais
}

\author{
par Martin Herden, université Laval et \\ Université libre de Berlin
}

Le terme stream of consciousness appartient d'abord au vocabulaire de la psychologie, où il désigne une qualité de la conscience, laquelle n'est jamais statique mais toujours en état de mouvement. En transposant cette métaphore à la littérature, on parle donc de la vie psychique d'un personnage, représentée dans certaines auvres littéraires. Robert Humphreyl définit ainsi le genre stream of consciousness novel par son contenu principal, soit la conscience d'un ou de plusieurs personnages. Il précise qu'il s'agit d'une conscience à un niveau inférieur (ou antérieur) à l'articulation dans la langue.

La forme littéraire la plus répandue pour présenter un stream of consciousness est le monologue intérieur. Dans son sens large, ce texte peut désigner des pensées, des impressions, des sentiments, etc. qu'un personnage n'articule pas, mais qui sont néanmoins présentés dans un texte. Pour préciser les particularités de cette technique littéraire, Dorrit Cohn propose de la nommer monologue intérieur autonome parce qu'elle place [...] d'emblée le lecteur dans l'intimité d'une conscience verbalisant en silence ses préoccupations immédiates ${ }^{2}$. L'essentiel dans ce procédé est l'absence de contexte, dans lequel le narrateur pourrait donner des indications sur la situation et le personnage. Cette absence de contexte implique que le monologue autonome n'est possible qu'à la première personne puisque le narrateur s'efface et que le personnage se substitue à lui. L'exemple canonique pour illustrer cette technique est le monologue de Molly dans Ulysses de James Joyce.

Dans le récit hétérodiégétique, ainsi nommé parce que le narrateur est absent de l'histoire qu'il raconte, Cohn distingue trois techniques de base ${ }^{3}$. La première, le psycho-récit, correspond à ce que Humphrey appelait la description omnisciente. La deuxième, le monologue rapporté, se distingue du monologue autonome par la présence d'un contexte dans lequel le narrateur hétérodiégétique cite de différentes manières le monologue intérieur d'un personnage. La troisième

1 Robert Humphrey, Stream of Consciousness in the Modern Novel, Berkeley, University of California Press, 1954, 127 p.

2 Dorrit Cohn, la Transparence intérieure, Paris, Seuil, 1981, p. 250.

3 Ibid., p. 25 et ss. 
technique est le monologue narrativisé. Monologue narrativisé (narrated monologue) est une traduction du terme allemand erlebte Rede qui couvre un champ conceptuel beaucoup plus large, et a été traduit en français par style indirect libre. Pour situer le style indirect libre, Cohn l'oppose à la fois au discours direct et au discours indirect. Il se situe entre les deux parce qu'il partage, avec le discours indirect, la troisième personne pour désigner le personnage et les transpositions des temps verbaux et, avec le discours direct, la structure de la phrase (exclamations, répétitions, etc.). Néanmoins, il se distingue clairement de ces deux discours par l'absence du verbe déclaratif (par exemple pensait-elle).

Le style indirect libre équivaut au monologue narrativisé lorsqu'il cherche à rendre la vie intérieure d'un personnage. Une des caractéristiques de cette technique est la confusion entre narrateur et personnage. En conséquence, il est souvent impossible de décider si un segment donné, phrase, paragraphe ou même page entière, doit être attribué à l'un ou à l'autre. On verra plus loin comment cette confusion peut être exploitée.

Les deux textes analysés sont le Sourd dans la ville de Marie-Claire Blais et le deuxième chapitre du roman The Sound and the Fury de William Faulkner, soit le monologue de Quentin. Ce choix est motivé par plusieurs facteurs. Les deux auteurs utilisent les techniques du stream of consciousness. Sous cet aspect, il est possible de comparer deux narrations ultérieures, écrites au passé, dont l'une, celle de Blais, est présentée par un narrateur hétérodiégétique, et l'autre, le monologue de Quentin, par un narrateur autodiégétique. Malgré cette divergence, on peut parler de monologue intérieur au sens large dans les deux récits.

D'une part, il y a donc le monologue unique de Quentin et, d'autre part, il y a les multiples monologues intérieurs du roman de Marie-Claire Blais. Pour expliquer cette disproportion dans l'analyse, il faut regarder de plus près le rôle de Florence dans le Sourd dans la ville. Elle n'est qu'un personnage parmi d'autres, mais sa perspective domine le récit entier. Son rôle par rapport aux autres personnages est indiqué dans cet extrait:

[...] le vieux Tim ne pouvait pas dire si c'était une certitude, ses mots n'étaient pas clairs, définis, car il ne parlait que par blasphèmes, telle était sa haine ou son amour, [...] mais Florence éprouvait ces sentiments à sa place, car elle était malheureuse mais lucide et cette flamme de la conscience était une chose si froide $[. .$.$] (p. 51) 4$

On risque ainsi de confondre Florence avec la narratrice du roman. Cet aspect est renforcé par le fait que le récit, comme celui de Quentin, s'arrête avec la mort du personnage central. De plus, les deux héros, marqués par une éducation humaniste, sont les seuls qui écrivent. À plusieurs reprises, Quentin mentionne les lettres qu'il vient d'écrire; les bagages de Florence se limitent à une

4 Les citations des deux ouvres à l'étude se réferent aux éditions suivantes: MarieClaire Blais, le Sourd dans la ville, Montréal, Stanké, 1979, 274 p.; William Faulkner, The Sound and the Fury, New York, Vintage Books, 1946, 429 p. 
lourde valise: [...] remplie de lettres anciennes, on eût dit que ce poids des mots écrits et livrés, pendant une vie, l'aiderait à partir $[. ..](\mathrm{p} .30)^{5}$

Après avoir indiqué le statut privilégié de Florence, il est plus facile de s'interroger sur la relation entre les différents monologues. On peut considérer que les monologues dans le Sourd dans la ville sont subordonnés à un stream of consciousness, c'est-à-dire que les monologues de Mike, de Tim, de Berthe, etc. sont des variantes du monologue intérieur de Florence - Florence comprise dans son rôle ambigu de personnage-narratrice. En ce sens, l'errance solitaire de Florence est représentée différemment par les autres personnages.

L'amour avait rendu possible un bonheur familial et un univers qui semblait être cohérent. Au moment de la remémoration, lorsqu'elle est dans l'escalier, elle revit la rupture avec son mari et on constate ses effets: le monde a perdu sa cohérence et le bonheur passé, maintenant qualifié d'illusoire, est remplacé par le désespoir et l'angoisse.

Cette situation est reproduite - sans que cela dépasse l'énoncé général du roman qui ne cesse de déplorer la misère de ce monde - dans celle de Gloria dont le mari est mort. Son bonheur est, dans le passé, la gloire de son mari, et, dans un avenir illusoire, le voyage avec son fils, en moto, vers une Californie mythique. Le monologue de Berthe, personnage qui souffre de sa solitude après avoir rompu avec sa famille, en est une autre variante; de même celui de Tim qui a tué son double, son chien, ou encore Tim qui rêve de l'Irlande, son pays d'origine duquel il sera à jamais séparé.

En limitant l'analyse au deuxième chapitre du roman de Faulkner, plusieurs parallèles apparaissent. D'abord, on voit que les deux récits sont dominés par leur fin, le suicide du personnage central. Au niveau socio-culturel, ces deux personnages se ressemblent, parce qu'ils sont plus cultivés, plus instruits que leur entourage et ne vivent pas dans le même milieu. Dans la structure du texte, ils ont une fonction commune: ils posent leur regard sur un enfant handicapé. Florence voit Mike, le sourd dans la ville, et Quentin reste sous l'effet du leitmotiv Benjy bellowing.

\section{Les perceptions sensorielles}

Avant de préciser la fonction des deux enfants handicapés, posons la question des perceptions sensorielles par lesquelles les deux textes sont dominés. Tandis que le monologue de Quentin est marqué par des impressions sensuelles très fortes, notamment par les odeurs et les sons, le roman de MarieClaire Blais est dominé par le visuel. Il faut d'abord insister sur la riche sonorité du texte original de Faulkner que la traduction française ne rend que fort mal. Il

5 On peut noter en passant que ce personnage correspond à l'idée que Marie-Claire Blais se faisait du devoir éthique de l'écrivain aux prises avec une société déplorable: Il ['écrivain] contient beaucoup de vies en lui, dans son témoignage. Puis il parle pour les autres, il est le visionnaire des autres, le visionnaire de son temps. (Entrevue accordée à Voix \& images, vol. VIII, no 2, hiver 1983, p. 191) 
est difficile de faire équivaloir une sonorité riche comme Benjy bellowing avec sa traduction le hurlement de Benjy.

Le monologue de Quentin commence avec un elément visuel: When the shadow of the sash appeared on the curtains [...] (p. 93). L'ombre de la fenêtre à guillotine apparait, indique l'heure et annonce la mort du personnage. Pour ne pas voir cette ombre qui va descendre, il se tourne dans son lit. De même, Quentin essaie pendant toute la journée de ne pas voir l'heure qu'il est. Il détruit les aiguilles de sa montre et le son de cloche persiste et est perçu comme une hantise.

Les impressions sensuelles de Quentin permettent souvent des transitions d'un niveau temporel à un autre. Ainsi, l'odeur du honeysuckle (chèvrefeuille) - peu importe s'il est effectivement senti ou remémoré- est d'une telle force qu'il perturbe les pensées du personnage. Il évoque Caddy et la sexualité. Presque toujours, il porte des connotations négatives, parce que c'est l'éveil de la sexualité qui a séparé le frère et la sœur et qui a mis fin à leur enfance commune. Quentin perçoit sa propre sexualité comme un péché. Il l'associe à la perte de la virginité de Caddy, ce qui le mène à avouer un inceste, jamais consommé, avec sa sœur.

À plusieurs reprises, c'est la présence du sang qui permet l'enchainement de deux scènes. Malgré la présence du mot blood dans tout le texte, la couleur n'est jamais évoquée dans ce contexte. Dans le passage suivant, le sang est l'élément qui lie la scène remémorée - Quentin et Caddy après leur pacte de suicide - à la lutte entre Quentin et Gerald Bland. On voit de quelle manière le regard est nié et comment Quentin perçoit les événements:

do you love him Caddy

do I what

she looked at me then everything emptied out of her eyes and they

looked like the eyes in the statues blanc and unseeing and serene

put your hand against my throat

she took my hand and held it flat against her throat

now say his name

Dalton Ames

I felt the first surge of blood there it surged in strong accelerating beats

say it again (p. 203)

Cette forte sensualité marque tout le monologue de Quentin et met en scène les éléments de son inconscient qui déclenchent la crise et le suicide final. Les problèmes causés par la sexualité et la perte de Caddy, le bonheur de son enfance, sont transposés au niveau de la conscience, où ils apparaissent dans les réflexions de Quentin sur la virginité, l'honneur et la fatalité du temps. Un des motifs les plus fréquents, Benjy bellowing, condense le conflit de base. La mise en relief de ce leitmotiv permet de lire tout le monologue comme l'interprétation d'une impression sonore qui est le noyau du texte.

Dans le Sourd dans la ville, les impressions sonores sont très faibles: même la détonation du revolver à la fin est décrite comme un bruit sourd. 
Néanmoins, on trouve un élément qui correspond au leitmotiv acoustique de Faulkner: le Cri d'Edvard Munch. Cette impression visuelle semble engendrer tout le roman et on peut dire qu'il s'agit de la transposition d'un tableau en écriture.

En regardant le tableau de Munch, on remarque les couleurs malsaines, un peu sales, qu'on retrouve chez Marie-Claire Blais dans la description de l'Hôtel des Voyageurs. Puis il y a le mouvement chaotique du paysage, évoquant, si on veut, un tremblement de terre, contrasté par la diagonale, le pont. Cette diagonale introduit un mouvement, une vitesse angoissante que Marie-Claire Blais a réalisés dans le rythme de son texte, en noyant les différents monologues dans un fleuve langagier qui coule à une trop grande vitesse. L'ombre de Tim et sa version abstraite, la mort finale qui jette son ombre sur le texte entier, semblent être inspirées par l'ombre du personnage central de Munch qui est encore plus prononcée dans un autre de ses tableaux intitulé Adolescence. Un dernier détail du tableau, l'enfant qui presse les mains contre ses oreilles pour ne pas entendre le cri de la nature, peut être repéré dans la surdité de Mike et aussi dans l'attitude de Florence qui reste sourde à la demande des femmes qui cherchent à créer un contact avec elle.

Quelques autres motifs comme les lilas blancs, les rosiers, le jardinier au milieu de ses fleurs, etc. semblent être inspirés par certains tableaux impressionnistes. La prédominance des perceptions visuelles dans le Sourd dans la ville est facile à démontrer, puisque les leitmotive sont de l'ordre du visuel. Il y a des motifs simples, par exemple les lilas blancs ou Berthe avec le chat, mais aussi des constellations plus complexes qui reviennent plusieurs fois, comme la scène qui montre Mike, sous un arbre, jouant avec sa petite scur et regardant les deux Tim (l'homme et le chien). À côté des motifs stables comme les lilas blancs, on trouve des motifs qui sont variés, interprétés de différentes manières. Ainsi, le pansement de Mike est d'abord introduit d'une manière naturaliste, lié à la maladie, à la tumeur. Ensuite, cette même image évoque un tableau de Munch (l'Enfant malade), une représentation du Christ et puis l'archange Gabriel.

Malgré cette multitude d'éléments visuels, un regard domine le texte: celui de Florence. Il ressemble étrangement à sa manière de regarder des tableaux. Par exemple, le regard qu'elle pose sur Mike, le sourd dans le bar, ne se distingue guère du regard qu'elle pose sur le tableau de Munch. Il n'y a pas de communication entre Florence et Mike - tout comme il n'y en a pas entre Quentin et Benjy - parce que Mike est sourd et ne parle presque.pas. Son cri de douleur reste un cri muet comme celui du tableau de Munch et c'est justement ce cri qui réclame le discours muet qu'est le monologue intérieur.

\section{La temporalité chez Faulkner}

Le lecteur de The Sound and the Fury se voit confronté à un problème de compréhension qui est de l'ordre de la temporalité. Au début du roman, on se demande constamment de quel événement parle le narrateur. Peu à peu, cette confusion disparaît et, à la fin du livre, le lecteur est en mesure de situer les différents épisodes. En retraçant l'histoire dans cet ordre nouveau, on se rend compte, 
comme Sartre l'a remarqué6 ${ }^{6}$ qu'on est en train de raconter une autre histoire. Voyons donc de plus près la situation temporelle du monologue de Quentin.

Dès les premières lignes du chapitre, le lecteur intègre la conscience de Quentin; il s'agit d'un monologue autonome. En définissant cette modalité narrative, Cohn écrit que la conscience verbalise ses préoccupations immédiates ${ }^{7}$. On obtient ainsi un niveau temporel de référence qui est le présent de la conscience et qui, dans l'exemple classique du monologue de Molly Bloom, coïncide avec le moment de l'énonciation du monologue. Dans le monologue de Quentin, le temps ou plutôt le lieu temporel de la narration est différent, puisqu'il s'agit d'une narration ultérieure dans laquelle tout est raconté au passé. La seule préoccupation immédiate de Quentin-narrateur est la remémoration des scènes du passé.

À cause de cette différence, Cohn propose de parler d'un monologue remémoratif 8 qui est une variante du monologue autonome. Une de ses caractéristiques est la confusion des différents niveaux temporels qui résulte de l'emploi du même temps grammatical pour désigner les différents événements. Tandis que le monologue remémoratif présente les événements dans l'ordre de leur remémoration, un narrateur peut aussi les présenter dans l'ordre chronologique. Cette deuxième technique qu'on pourrait appeler monologue autobiographique est utilisée dans le texte de Faulkner pour désigner les événements de la dernière journée de Quentin. Ainsi, le monologue autobiographique qui progresse en parfait ordre chronologique, du début à la fin du chapitre, relie tout le monologue et peut fonctionner comme un niveau temporel de référence.

Le lecteur qui essaie de s'orienter de cette manière est tenté d'identifier ce niveau temporel avec le présent. Une telle confusion peut aussi amener à parler de «scènes réelles» pour désigner les événements de cette dernière journée:

[...] I found the gasoline in Shreve's room and spread the vest on the table where it would be flat, and opened the gasoline.

the first car in town a girl Girl that's what Jason couldn't bear smell of gasoline making him sick [...] (p. 213)

Dans cette transition, Quentin se souvient de Caddy dans sa voiture, quelques jours avant le mariage, et de Jason qui, en général, ne supporte pas l'odeur de l'essence. Ces souvenirs semblent surgir dans la scène «réelle», au moment où Quentin ouvre le bidon d'esence. Ainsi, l'odeur est censée déclencher les souvenirs et on peut supposer que le monologue se situe dans le temps du personnage. Sinon, il faudrait dire que le narrateur, longtemps après l'incident, reproduit l'association que le personnage faisait à ce moment.

Ailleurs dans le texte, il semble évident que la scène est évoquée après l'événement rapporté dans le monologue autobiographique. Quand Quentin se bat

6 Jean-Paul Sartre, «A propos de le Bruit et la fureur. La temporalité chez Faulkner», Situations I, Paris, Gallimard, 1947, p. 65.

7 Dorrit Cohn, op. cit., p. 250.

8 Ibid., p. 279 et ss. 
avec Gerald Bland, la scène est évoquée après coup, à travers les commentaires de Shreve. Au moment où cet événement est censé se produire, il y a une ellipse temporelle complète. Des souvenirs antérieurs, la scène du pacte de suicide avec Caddy et la confrontation entre Quentin et Dalton Ames, sont superposés et remplacent l'évocation de la scène «réelle». Il est difficile de croire que cette superposition de deux scènes se produit dans la conscience du personnage et on l'attribue donc à la conscience du narrateur qui évoque tout le chapitre au passé.

La position temporelle de la narration n'est pas très claire, mais à cause du temps des verbes, elle doit être située après le dernier événement du récit et donc après le moment où Quentin quitte la chambre pour se suicider. Quentinnarrateur raconte donc l'histoire juste avant sa mort, au moment même de mourir ou, comme Sartre l'affirme9, il est déjà mort quand il commence à remuer ses souvenirs.

Le monologue de Quentin est caractérisé par ses ruptures temporelles; ainsi Faulkner nous présente un personnage déchiré. Malgré cela, le texte lui-même demeure très cohérent puisque, dans les différents niveaux temporels, les mêmes obsessions reviennent. Les motifs renvoient en général aux deux grands thèmes du chapitre - la perception du temps et la perte de Caddy - et créent un réseau d'images qui lie les différents événements. Les pages 154 et 155 sont particulièrement riches en images temporelles. Après l'évocation de souvenirs antérieurs, en italiques, un chariot traîné par un cheval blanc apparaît brusquement dans le monologue autobiographique. Il fait appel aux mêmes métaphores, désignant un mouvement figé, que celles que l'auteur utilise pour désigner le temps qui passe:

[...] motionless in the fork, in the flickering shade [...] neatly rapid like the motions of a lady doing embroidery, diminishing without progress like a figure on a treadmill being drawn rapidly off-stage (p. 154).

Cette description produit un effet d'irréalité, renforcé par le fait qu'on peut voir dans l'apparition du chariot l'illustration des pensées qui la précèdent:

Man the sum of his climatic experiences Father said. Man the sum of what have you. A problem in impure properties carried tediously to an unvarying nil: stalemate of dust and desire. But now I know I'm dead I tell you.

Then why must you listen we can go away you and Benjy and me where nobody knows us where The buggy was drawn by a white horse $[. .$.$] (p. 154)$

L'irréalité de cette apparition dans le monologue autobiographique est encore renforcée quand on se souvient que le même motif est évoqué quelques pages plus haut, au moment où Quentin parle avec les trois garçons, leur demandant ce qu'ils feraient s'ils attrapaient le poisson: 
They all talked at once, their voices insistent and contradictory and impatient, making of unreality a possibility, then a probability, then an incontrovertible fact, as people will when their desires become words.

"I' $d$ buy a horse and wagon» the second said. [...] it was as though he had captured the fish and bought his horse and wagon [...] (p. 146)

La «réalité» des scènes du monologue autobiographique n'est donc qu'une illusion. Le même effet de conte de fées est produit quand Quentin entre dans la boulangerie où il rencontre la petite fille italienne. Dans les souvenirs antérieurs, en italiques, il est question de Caddy qui vient de perdre sa virginité et du hurlement de Benjy. La voix de Benjy est décrite, juste avant, comme le mouvement du chariot tiré par un cheval blanc. La scène «réelle» - l'entrée dans la boulangerie - semble être le résultat de la scène remémorée, dominée par Benjy bellowing:

\section{$[$...] in silence bellowing}

When you opened the door a bell tinkled [...] (p. 155)

Ici, l'association sonore bellowing - a bell engendre la suite du texte ${ }^{10}$. Au niveau thématique, la transition est réalisée par les références à la perception du temps et par les motifs. Dans l'ensemble du texte, les cloches évoquent pour Quentin le mouvement du temps, le moment déterminé du suicide qui approche et le mariage de Caddy. Le hurlement de Benjy est la plus forte expression de la douleur causée par la perte de Caddy. En plus, on remarque que Quentin retrouve dans la boulangerie un bonheur du passé, de l'enfance:

[...] the door opened upon the recent warm scent of baking; a little dirty child with eyes like a toy bear's and two patent-leather pig-tails.

«Hello sister.» Her face was like a cup of milk [...] (p. 155)

L'expression sensorielle de Quentin-personnage est dominée par l'odeur, tandis que Quentin-narrateur, en présentant la petite fille, renvoie aussi à d'autres motifs du texte. L'épithète little dirty child sert en général à évoquer Nathalie et la sexualité refoulée. En plus, on peut y voir une référence au muddy drawers de Caddy 11 et donc à la perte de sa virginité. Quand la petite fille est appelée sister, on y voit d'abord une identification avec Caddy, puis une référence au jargon des Noirs et finalement saint François qui appelait la mort Little Sister Death.

L'analyse de la temporalité dans le monologue de Quentin dévoile plusieurs particularités du texte. D'abord, dans le monologue remémoratif, le présent de l'énonciation est vidé et le narrateur ne rapporte pas les expériences qu'il vit

10 Cette manière de lier les différents niveaux temporels par une association sonore est fréquente dans le texte; par exemple à la page 124 où elle est produite entre trois termes: the belly of my shadow - bells - Benjy bellowing.

11 Cette association sera exploitée un peu plus tard, à la page 163. 
immédiatement. Puis le présent du personnage, celui du monologue autobiographique, est brisé par les irruptions violentes du passé. Quentin perçoit son présent comme une répétition fatale du passé (par exemple dans les superpositions Caddy - Nathalie - la fille italienne; ou Dalton Ames - Herbert Head - Gerald Bland). Il est hanté par le temps qui progresse sans s'ouvrir vers un avenir possible.

Faulkner a créé un personnage déchiré par ces ruptures, mais l'ensemble du texte est plutôt cohérent. Comme les obsessions de Quentin, les motifs se répètent dans des contextes différents. Cette multitude de renvois produit un texte surchargé de significations et en même temps clos, puisque toutes les associations se font à l'intérieur de la conscience du personnage. Les écrivains du nouveau roman ont atteint un effet comparable par la multiplication de miroirs dans lesquels les différents reflets renvoient toujours aux mêmes objets. Dans le monologue de Quentin, cette clôture du texte met en évidence le caractère obsessionnel des souvenirs et accentue la fatalité dans la suite des événements: le suicide inévitable de Quentin, la perte irrémédiable de Caddy, de l'enfance...

La temporalité dans le roman de Blais et les questions de focalisation chez Faulkner

Avant d'analyser la focalisation dans le Sourd dans la ville, considérons le rôle du temps dans ce livre. On retrouve, dans la situation du personnage principal, un parallèle avec le roman de Faulkner: comme Quentin, Florence vit une rupture temporelle. Elle n'est pas séparée de son enfance, de son innocence, mais de son bonheur familial. Dans plusieurs de ses monologues, elle évoque ce passé heureux en parlant de son amour, de son mari, de son enfant, ou en faisant référence aux peintres impressionnistes. Comme Quentin, elle vit un présent marqué par le désespoir et menant au suicide. Dans les deux cas, la mort, en dernière instance, jette son ombre sur tout le récit. En cherchant les raisons qui mènent au suicide, on remarque plusieurs ressemblances dans les réflexions «nihilistes» des protagonistes.

Marie-Claire Blais introduit une confusion des niveaux temporels par l'utilisation abondante de l'imparfait. Cet imparfait indique d'abord qu'il s'agit plus de dépeindre une situation que de rapporter des événements. L'utilisation de ce temps est favorisée par le fait que la langue française permet l'imparfait aussi bien dans le discours indirect que dans le style indirect libre - ce qui n'est pas le cas, par exemple, en allemand 12 .

Pour comprendre le conflit essentiel, le lecteur n'est pas obligé de mettre les événements dans un ordre chronologique. Comme dans le cas du monologue de Quentin, le récit des événements de la dernière journée de Florence constitue un niveau temporel de référence qui peut faciliter la lecture. Ainsi, au niveau de la temporalité, Blais arrive à produire l'effet d'un glissement ou d'une imprécision temporelle qui s'accorde avec le flou qui caractérise l'aspect visuel du roman.

12 Guenter Steinberg, Erlebte Rede. Thre Eigenarten und Ihre Formen in neuerer deutscher, französischer und englischer Erzählliteratur, Göppingen, Kümmerle, $1971,476 \mathrm{p}$. 
Une analyse de la focalisation dans The Sound and the Fury est certainement faisable, mais dans la partie du roman qui nous intéresse ici - le deuxième chapitre - , la question essentielle est de savoir si les transitions, les associations se réalisent au niveau de la conscience de Quentin-personnage ou de Quentin-narrateur. Il s'agit donc d'un problème de temporalité ou, si on veut, de la question de Genette: qui parle ${ }^{13}$, mais pas d'un problème de focalisation.

Les ruptures temporelles du texte de Faulkner et les changements de focalisation dans le roman de Marie-Claire Blais ont un statut comparable en ce qu'ils posent tous deux le problème majeur de la compréhension. Ils dérangent le lecteur parce qu'ils sont inhabituels et donc particuliers à l'écriture de l'œuvre.

\section{La focalisation chez Blais}

La focalisation instable dans le Sourd dans la ville crée une difficulté de compréhension qui marque tout le texte. Pour pouvoir distinguer les différents personnages et monologues, le lecteur est obligé de se demander constamment quelle est la perspective narrative dans un segment donné.

Étant donné que le roman est raconté par un narrateur hétérodiégétique, il est exclu de parler de monologue intérieur dans le sens précis de monologue autonome. Pour présenter le stream of consciousness - métaphore qui décrit très bien l'ensemble du texte - Marie-Claire Blais utilise les trois techniques de base définies par Cohn: le psycho-récit, le monologue rapporté et le monologue narrativisé. La troisième de ces techniques est particulièrement intéressante, parce qu'elle favorise la fusion entre narrateur et personnage et le glissement de l'extérieur (actions, descriptions d'un personnage, etc.) à l'intérieur d'une conscience évoquant un monologue intérieur. Voyons ces effets dans le texte:

\section{[...] assise dans les marches de l' escalier elle [Florence] attendit, qui étaient-ils oui qui étaient-ils ceux qui vivaient ici, ici ou ailleurs sur cette terre ou elle achevait son exil, appuyée contre la rampe de l'escalier, elle écoutait, observait, se taisait, toute calme [...] (p. 49)}

On voit très bien quelques caractéristiques du monologue narrativisé, qui débute à qui étaient-ils [...] et va jusqu'à appuyée contre la rampe, l'absence du verbe déclaratif, le caractère émotionnel de la phrase qui ressemble à un discours direct et l'emploi de l'adverbe déictique ici au lieu de là qui serait exigé dans le discours indirect et dans la description par un narrateur hétérodiégétique en général. La confusion entre narrateur et personnage est accompagnée d'une confusion entre les personnages qui focalisent. Un auteur qui représente la vie psychique d'un personnage imite, dans une certaine mesure, un stream of consciousness; ce qui explique l'instabilité de la focalisation sur des objets. L'instabilité de l'instance focalisante est, en revanche, une particularité du roman de Marie-Claire Blais ${ }^{14}$. Dans le texte, les différents monologues ne sont pas liés par une action extérieure. Il s'agit plutôt d'une juxtaposition de scènes

13 Gérard Genette, Figures III, Paris, Seuil, 1972, p. 203.

14 Cf. Mieke Bal, Narratologie, Paris, Klincksieck, 1977, 199 p. 
et de perspectives. Ces changements de focalisateur sont indiqués par une multitude de formules stéréotypées, comme pensait-elle, se disait Mike, etc. Souvent, un passage qui commence par Mike se demandait [...] se termine par [...] pensait Florence et à l'intérieur de cette séquence, les verbes peuvent se référer aux deux. Ainsi, il devient impossible de déterminer exactement où le monologue de l'un se termine et où commence celui de l'autre.

Un autre type de changement de focalisation permet de saisir le statut de quasi-narrateur de Florence. Quand elle focalise sur un personnage - par exemple Mike -, celui-ci, à son tour, commence à focaliser sur quelque chose; ainsi, le monologue intérieur de Mike est marqué par le ton et par le vocabulaire de Florence. La fusion des différents monologues intérieurs se réalise donc aussi bien dans la perception du lecteur qui n'arrive pas à distinguer les focalisateurs qu'au niveau de la conscience de Florence.

\section{Structure romanesque et matérialité des œuvres}

Le roman de Faulkner est structuré de différentes manières. Au niveau thématique, l'action extérieure - la suite des événements dans le monologue autobiographique -, l'ordre des scènes remémorées et la répétition obsessionnelle de certains motifs, permettent au lecteur de structurer le texte. Une autre manière d'orienter la lecture, la présentation matérielle du texte, est largement exploitée par l'auteur. En analysant cet aspect, on parlera surtout des effets de rythme qui, déjà au niveau de la phrase, est valorisé par la sonorité des mots choisis.

Le monologue intérieur de Quentin est imprimé dans deux caractères typographiques différents. Les italiques (reproduits en caractères gras ici) indiquent toujours un changement de niveau temporel dans la conscience du personnage et facilitent ainsi la compréhension du roman. De plus, ils mettent en scène la conscience déchirée et le caractère obsessionnel de ses souvenirs. Souvent, les passages en italiques sont introduits violemment dans le monologue autobiographique et, en accentuant les ruptures, marquent l'ensemble du texte à un point tel qu'il semble absurde d'utiliser la métaphore stream of consciousness pour le décrire.

Outre les italiques, Faulkner utilise d'autres moyens pour distinguer quelques grandes unités du texte, notamment la présence ou l'absence de ponctuation, de guillemets, de majuscules et de paragraphes. Pour saisir les changements de rythme provoqués par la combinaison de ces moyens, on peut maintenant confronter certains passages d'une grande dissemblance.

Le monologue autobiographique est souvent caractérisé par la structure syntaxique des phrases. L'écrivain obéit strictement aux lois de la grammaire, sépare des unités de sens par des paragraphes, signale un dialogue rapporté en discours direct par des guillemets, etc. En lisant un tel passage, le lecteur reproduit la scène «réelle», par exemple un dialogue, d'une manière réaliste, sans être contrarié par des ruptures ou des changements de rythme inhabituels.

Dans la scène du pacte de suicide avec Caddy et la rencontre avec Dalton Ames (p. 186-203), l'auteur prend davantage de liberté dans la présentation du 
texte. On remarque d'abord l'absence totale de ponctuation et la disposition en vers du texte. Toute cette partie est marquée par une forte sensualité. Même les actions de Quentin qui, souvent, représentent des ruptures dans le stream of consciousness, sont intégrées dans le flot de langage. Par l'absence de ponctuation, l'auteur insiste moins sur la structure logique des phrases et valorise la qualité rythmique du texte. Après les ruptures violentes du début, la musicalité de ce passage provoque un plaisir qui, dans la diégèse, renvoie aux sentiments des personnages. D'ailleurs, on remarque que la scène entière est imprimée en caractères romains. L'absence d'italiques et donc de rupture temporelle est motivée par le fait que Quentin arrive à échapper au temps, au présent catastrophique des demières heures de sa vie. Sa conscience est entièrement absorbée par les souvenirs de l'enfance, marqués par la présence sensuelle de sa sœur.

À plusieurs reprises, un alinéa coupe une phrase en plein milieu. Vers la fin du monologue de Quentin, on remarque au contraire plusieurs pages sans paragraphe, sans ponctuation et même sans majuscule. Le rythme accélère, renforcé par les enchaînements réduits au minimum (and I... and he... and I). Cette accélération provoque un sentiment d'angoisse chez le lecteur au moment où Quentin semble avoir peur de la mort qui s'approche. À la dernière page, le texte retrouve son rythme normal et on observe la correspondance entre l'ordre parfait dans la présentation matérielle du texte et la pédanterie avec laquelle Quentin accomplit les demiers gestes de sa vie.

Dans le Sourd dans la ville, Marie-Claire Blais exploite beaucoup moins les possibilités de la disposition différenciée du texte. Le roman est écrit dans un seul paragraphe, contrairement au monologue de Quentin. Dans ce texte-fleuve, les points qui pourraient définitivement marquer la fin d'une phrase sont négligeables. Même s'ils apparaissent avec une certaine régularité (à peu près toutes les deux pages), ils ne marquent pas de césures importantes. Les virgules servent d'une part à distinguer les unités syntaxiques et d'autre part à rythmer le flot de langage.

Dès les premières lignes, le lecteur, entraîné dans le mouvement du texte, est obligé d'accélérer sa lecture. Après quelques pages, cette vitesse de lecture impose un choix au lecteur: ou bien il essaie de structurer le texte en imaginant la ponctuation négligée par l'auteure, ou bien il se laisse emporter par le rythme. Cette deuxième possibilité, qui semble être la seule solution satisfaisante, mène à une lecture plutôt superficielle puisqu'elle empêche de préciser les situations syntaxiques ambiguès. Elle peut être justifiée par le roman lui-même qui se présente comme le grand tableau d'une situation générale, rassemblant plusieurs perspectives et non l'analyse approfondie de cette situation.

Le rythme de ce texte provoque des sentiments ambigus: il peut épuiser le lecteur, entraîner l'enfoncement dans une angoissante incertitude - ce qui correspond à un des énoncés principaux du roman - et, en même temps, il propose le bonheur de se perdre dans un flot langagier ${ }^{15}$. Ainsi, dans les vingt

15 Cette ambiguïté n'est pas rendue dans le film que Mireille Dansereau a réalisé d'après le roman. Tandis que le lecteur est entraîné dans la vitesse angoissante du 
dernières pages du livre, on remarque une liquéfaction du style (le rythme devient plus agréable, plus régulier, les oppositions sémantiques s'atténuent, etc.) qui rend la lecture plus agréable. Le suicide final, annoncé dès le début du texte, semble perdre ses connotations négatives et se transforme en délivrance.

Pour résumer, on peut dire que la fusion des différents monologues intérieurs dans un stream of consciousness, réalisée surtout dans le style indirect libre et les changements de focalisateurs, est soulignée par un rythme unificateur. L'homogénéité du texte provoque une ambiguité étonnante: la solitude essentielle des personnages, dont il est question dans le récit, est niée au niveau formel par une technique littéraire qui a noyé tous les monologues dans un seul fleuve langagier.

La représentation de la vie psychique des personnages était le point de départ de cette analyse. Au lieu de chercher des éléments identiques, il s'agissait plutôt d'opposer les deux textes afin de découvrir les particularités des techniques romanesques. En partant de quelques ressemblances, comme la situation de rupture, le suicide final, le regard sur un enfant handicapé et la narration ultérieure, on a pu constater un certain nombre de problèmes semblables au niveau de la conscience des personnages. Cependant, les techniques littéraires utilisées par les auteurs sont très différentes.

Le monologue de Quentin évoque un personnage déchiré par des ruptures temporelles qui marquent le texte et mettent en relief l'importance du temps dans la diégèse. Le "présent» du personnage dans le monologue autobiographique est vécu comme un mouvement fatal vers la mort, brisé par les irruptions violentes des scènes du passé. L'autre présent, celui de la narration, est vidé de toute expérience immédiate, sauf l'énonciation du monologue autonome, la remémoration. Les impressions sensorielles qui dominent la perception du personnage central, et donc l'ensemble du texte, sont les odeurs et les sons. La force de ces impressions peut accentuer les ruptures et permet l'évocation d'un bonheur et d'un désespoir très forts.

Tandis que Faulkner exploite une multitude de moyens techniques pour introduire des changements de rythme et pour varier la perception du texte, le roman de Marie-Claire Blais est marqué par une tonalité et un rythme plutôt uniformes. La métaphore du stream of consciousness convient particulièrement bien pour décrire le Sourd dans la ville puisque les différents monologues sont subordonnés à un monologue intérieur, celui de Florence, et forment ainsi un courant de conscience cohérent. Pour laisser une certaine ambiguïté au texte, l'auteure a introduit des glissements d'un focalisateur vers un autre et, en utilisant le style indirect libre, des glissements entre la description par un narrateur hétérodiégétique et le monologue intérieur d'un personnage.

rythme de Blais, le spectateur du film reste dans un état de contemplation. Il peut regarder les belles images de la misère sans être dérangé par la forme de l'œuvre - effet qu'auraient produit par exemple des changements de plans inopinés, plus violents. 
La prédominance des perceptions visuelles peut se résumer à un regard - le regard posé sur le Cri d'Edvard Munch. Ce tableau semble déclencher tout le roman et correspond en tant que noyau du texte, au leitmotiv acoustique Benjy bellowing de Faulkner. On découvre ainsi, au centre des deux œuvres, une douleur, ou plus précisément un cri de protestation, contre une situation intolérable. Dans les deux cas, ce cri est lié à un enfant handicapé - la surdité de Mike et l'incapacité de s'exprimer de Benjy - et trouve son expression littéraire dans les monologues intérieurs. Pour mieux saisir la fonction de ce cri, on pourrait comparer les deux romans à d'autres œuvres d'art qui valorisent le cri dans leur construction esthétique. Je pense au film India Song, de Marguerite Duras, dans lequel le seul moment où l'image et la bande sonore coïncident est celui où crie le vice-consul. 